\title{
Exploratory Whisking by Rat Is Not Phase Locked to the Hippocampal Theta Rhythm
}

\author{
Rune W. Berg, ${ }^{1}$ Diane Whitmer, ${ }^{2,3}$ and David Kleinfeld ${ }^{1,3,4}$ \\ ${ }^{1}$ Department of Physics, ${ }^{2}$ Division of Biological Sciences, ${ }^{3}$ Graduate Program in Computational Neurobiology, and ${ }^{4}$ Center for Theoretical Biological \\ Physics, University of California, San Diego, La Jolla, California 92093
}

The rat has a strong 6-9 Hz rhythm of electrical activity in the hippocampus, known as the theta rhythm. Exploratory whisking, i.e., the rhythmic movement of the rat's vibrissas to acquire tactile information, occurs within the same frequency range as the theta rhythm and provides a model system to examine the relationship between theta rhythm and active sensory movements. In particular, it has been postulated that these two rhythms are phase locked as a means to synchronize sensory and hippocampal processing. We tested this hypothesis in rats trained to whisk in air. Theta activity was measured via field electrodes in the hippocampus, and whisking was measured via the mystacial electromyogram. We calculated the spectral coherence between these two signals as a means to quantify the extent of phase locking. First, we found that the fraction of epochs with high coherence is not significantly greater than that expected by chance (seven of eight animals and as a population average). Second, we found that the trial-averaged coherence is low (coherence, $<0.1$ ) and, as an average across all animals, statistically insignificant. We further asked whether the strength of the theta rhythm correlated with that of whisking, independent of the lack of cycle-by-cycle coherence. We observe that the correlation is weak and insignificant (six of eight animals and as a population average). We conclude that there is no relationship between the whisking and theta rhythms, at least when animals whisk in air.

Key words: EMG; electromyogram; hippocampal function; vibrissa; whisker; hippocampus; motor; rat; movement; motion; motor activity; theta rhythm; oscillator; whisking

\section{Introduction}

It is a matter of conjecture that oscillatory electrical dynamics in populations of hippocampal and cortical neurons serve a functionally relevant role (Buzsaki and Draguhn, 2004). A large slowwave oscillation at $6-9 \mathrm{~Hz}$, known as the theta rhythm, is one of the most salient electrophysiological properties of the hippocampus and is readily observable in many species. Investigators have been trying to elucidate the functional role of this rhythm. Numerous behavioral studies have demonstrated the coexistence of hippocampal theta rhythm and various voluntary motor patterns, including locomotion, orienting, rearing (Vanderwolf, 1969; Whishaw and Vanderwolf, 1971), swimming (Whishaw and Vanderwolf, 1971; Whishaw and Schallert, 1977), and paradoxical sleep (Vanderwolf, 1969; Winson, 1974). Exploratory whisking is an active rhythmic movement of the rat's tactile sensory organs, the vibrissas, which occurs with the same frequency as the theta rhythm (Kleinfeld et al., 1999; Berg and Kleinfeld, 2003) and occurs as a pattern with sniffing and head movements during exploration of novel objects (Welker, 1964).

Received Jan. 16, 2006; revised April 7, 2006; accepted May 5, 2006.

This work was supported by grants from the Human Frontier Scientific Program (D.K.) and the National Science Foundation Integrative Graduate Education and Research Traineeship program. We thank Gyorgy Buzsaki, Adam Kepecs, Jason Ritt, and Nachum Ulanovsky for discussions and Beth Friedman for comments on a previous version of this manuscript.

Correspondence should be addressed to Dr. Rune W. Berg, Department of Medical Physiology, The PANUM Institute, Room 12.5.5, Blegdamsvej 3, DK-2200 Copenhagen, Denmark. E-mail: rune@mfi.ku.dk.

DOI:10.1523/JNEUROSCI.0190-06.2006

Copyright $\odot 2006$ Society for Neuroscience $\quad$ 0270-6474/06/266518-05\$15.00/0
Whisking can occur in the absence of the theta rhythm, as demonstrated in experiments in which the medial septum was lesioned to abolish the theta rhythm (Gray, 1971; Semba and Komisaruk, 1984). Although this establishes the independence of the oscillations, the precise relationship between theta rhythm and whisking is presently ambiguous. Komisaruk (1970) described a specific phase relationship between the hippocampal theta rhythm and whisking by rat. A similar claim holds for the relationship between the hippocampal theta rhythm and sniffing by hamsters (Macrides, 1975). In contrast to this result, Vanderwolf's (1969) simultaneous recordings of hippocampal rhythm and the mystacial electromyogram (EMG), a surrogate of whisking (Carvell et al., 1991; Berg and Kleinfeld, 2003), demonstrated that whisking co-occurs with the theta rhythm only when the rat is moving its head and that hippocampal activity in an otherwise immobile rat is slow and irregular. Whishaw and Vanderwolf (1971) further demonstrated that the theta rhythm is absent in the hippocampus when vibrissas movement and sniffing cooccur in the absence of other movement. Last, recordings from swimming rats suggest the absence of a one-to-one relationship between whisking and theta (Whishaw and Schallert, 1977). Thus, although past results point toward the presence of independent rhythmic generators for whisking and for the theta rhythm, there is a discordant view as to whether these generators can phase lock. The importance of this issue is that locking can increase the reliability of synaptic transmission. This may provide a natural and efficient means to link the acquisition of new sen- 
A

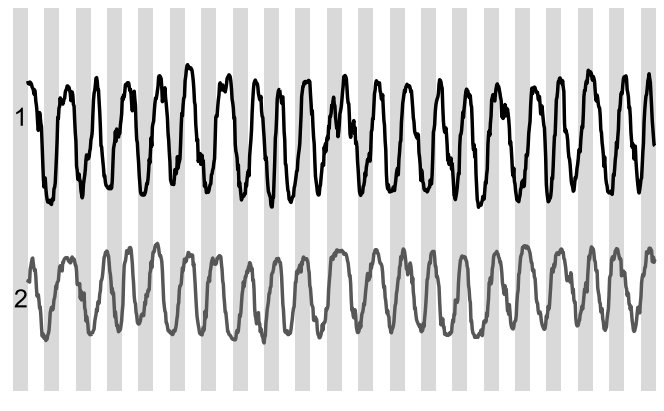

B

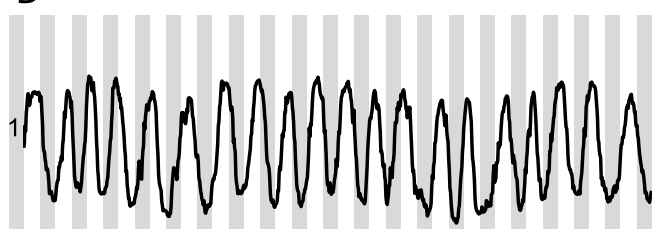

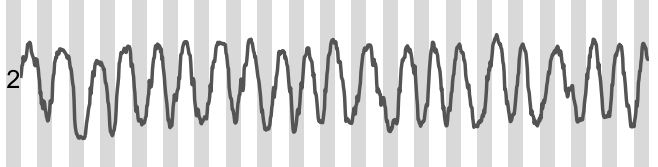
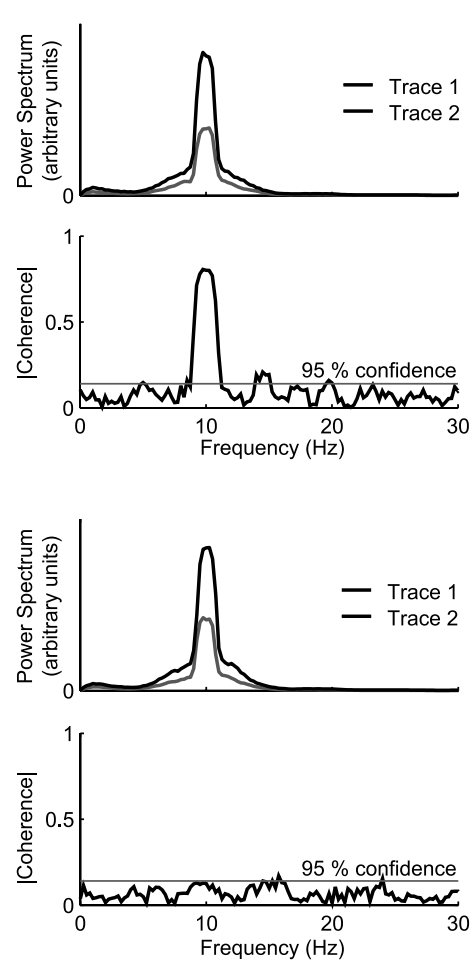

Figure 1. Illustration of coherence measurement between two rhythmic traces. The time series are based on simulated data that consist of sinusoids with added Gaussian amplitude noise, whose correlation time is $160 \mathrm{~ms}$ and SD is 30\% of the sinusoidal amplitude and added Gaussian phase noise, also with a $160 \mathrm{~ms}$ correlation time. The two time series have the same frequency but are modulated in frequency from trial to trial $(f=10.0 \pm 0.3 \mathrm{~Hz}$ ). $\boldsymbol{A}$, The two time series co-drift; the bands on the left are to guide the eye. The top right trace shows the two average power spectra of 50 samples, and the bottom right trace is the magnitude of average coherence, which is high in the region of substantial overlap of the power spectra. The gray horizontal line represents the magnitude for which the coherence is significant at $p<0.05$. $\boldsymbol{B}$, The two time series drift independently. The peaks in the two power spectra overlap substantially, as shown in the top right panel, but the magnitude of the coherence is low and insignificant at the overlap in frequency.

sory information, such as that gleaned from vibrissa touch, with memories of past experiences and locations, whose storage and recall is dependent on hippocampal activity (Treves and Rolls, 1994; Buzsaki, 2002; Hasselmo, 2005; Vertes, 2005).

Here we revisit the issue of coherence between whisking and the hippocampal theta rhythm. Our hypothesis is that the disparity in the early literature results largely from a visual rather than analytical comparison of the measured time series for whisking versus hippocampal activity. As a point of illustration, we consider the coherence between two rhythmic time series with substantial overlap in their spectral power estimates (Fig. $1 A, B$, top right). For the case in Figure $1 A$, the frequencies of the two signals co-drift and their spectral coherence, a measure of phase locking, is high. For the case in $B$, the spectral content of the two signals is identical, but they drift independently and thus are uncorrelated. The striking difference in coherence is unlikely to be found by visual comparison of the time series. In the present study, we calculate the coherence between the mystacial EMG and the hippocampal theta rhythm and quantify the statistical significance of this measure across multiple whisking epochs and a cohort of animals.

\section{Materials and Methods}

Our subjects were eight female Long-Evans rats, 200-300 g in mass. The mystacial EMG was recorded during whisking in air in free-ranging animals on an elevated runway, as described previously (Berg and Kleinfeld, 2003). These electrodes record motor unit activity from the intrinsic muscles. The signals from two electrodes that spanned mystacial pad were subtracted, highpass filtered, full-wave rectified, and low-pass filtered to form the measure of analysis, which we denote differential EMG ( $\nabla$ EMG); the gradient operator emphasizes that it is a spatially local measure of muscle activity. The local field potential (LFP) in hippocampus was recorded with a staggered triplet of Teflon-coated tungsten microwires, as described previously (Berg et al., 2005). The tips spanned a distance of 1 $\mathrm{mm}$, with the upper and lower electrodes in the dentate gyrus and the central electrode in the CA3 region. The locations of the electrodes were verified post hoc from Nissl-stained histological sections and by comparing the maximal amplitude of the theta rhythm with this in past studies (Robinson, 1980). The signals from three electrodes were subtracted to form the discrete second spatial derivative, denoted double-differential LFP $\left(\nabla^{2} \mathrm{LFP}\right)$, which is proportional to the radial contribution to the current source density. This electrical measurement provides a strictly local measure of hippocampal field activity, in this case current flow between the CA3 region and the dentate gyrus.

Each rat was gentled and acclimatized to the experimental environment over a period of 1 week before the surgical implantation of the electrodes. The animals recovered for $5 \mathrm{~d}$ after completion of surgery, after which data were subsequently collected daily for a period of $14 \mathrm{~d}$. Exploratory whisking was encouraged by the introduction of objects as the animal explored the runway and by the dimming of lights. In one-half of the animals, the infraorbital branch of the trigeminal nerve (IoN) was lesioned, as described previously (Berg and Kleinfeld, 2003), and data were collected for an additional $4 \mathrm{~d}$. The care and all aspects of the experimental manipulation of our animals were reviewed and approved by the Institutional Animal Care and Use Committee at University of California, San Diego.

Analysis. For each whisking epoch, of $T=2 \mathrm{~s}$, the spectral power of the mystacial $\nabla$ EMG and the hippocampal $\nabla^{2}$ LFP and the spectral coherence between these measures, denoted $C$, were calculated. The singletrial spectra were estimated as an average over $K=5$ independent estimates using the multitaper methods of Thomson (1982) (Percival and Walden, 1993). The half bandwidth is $\Delta f=(2 K-1) / T=1.5 \mathrm{~Hz}$. The center frequency for the whisking and theta rhythms were determined as the location of the peak in the respective power spectrum.

The confidence limit for the magnitude of the coherence across all frequencies was estimated from the following:

$$
|C|=\sqrt{1-P^{(N K-1)^{-1}}},
$$

in which $p=0.05$ represents the $95 \%$ confidence limit, $N$ is the number of trials or epochs, and NK is the number of degrees of freedom (Jarvis and Mitra, 2001). The trial-averaged coherence at the whisking frequency was calculated as the mean of the complex-valued coherence. Last, the correlation coefficient between the normalized amplitude of the power in the whisking spectrum and that in the hippocampal spectrum, was computed on an epoch-by-epoch basis for each animal. Confidence intervals on the correlation coefficients were computed as 2 SDs from the mean in a bootstrap histogram (1000 trials).

\section{Results}

We analyzed 885 whisking epochs across eight animals for which both rhythmic whisking and the hippocampal theta rhythm were 
within the range of $6-9 \mathrm{~Hz}$. In a fraction of the trials, $<10 \%$ of total, the theta rhythm had marginal power and those trials were discarded. For the example data of Figure 2 , the power spectra of the $\nabla E M G$ and the hippocampal $\nabla^{2}$ LFP were largely overlapping (Fig. $2 A, B$, top right panels). It is further evident that the two rhythms are locked to each other in some whisking epochs (Fig. 2A) but not in other epochs (Fig. $2 B$ ). The variability in the coherence across individual epochs illustrates how limited data sets can be used to support the results of Vanderwolf (1969) versus Komisaruk (1970). This variability does not depend on how closely the two spectral power peaks were aligned.

As a first means to establish the statistics of the coherence across individual epochs within each animal, the coherence between whisking and theta rhythm was computed for each epoch at the frequency of the peak in the power spectrum for whisking as well as that for the theta rhythm. The magnitude of coherence between two unrelated processes will satisfy the null hypothesis for $C=0.726$ (Eq. 1 with $N=1, K=5$, and $p=0.05$ ). The normalized distribution of coherence magnitudes across epochs for a representative animal (animal F9) shows that $<5 \%$ of the epochs exceed this level of coherence (Fig. 3A). This is particularly evident from the cumulative probability distribution, which crosses the $95 \%$ line before reaching the significance value of $C=0.726$ (Fig. $3 B$ ). As a population, the fraction of trials that had coherence with a magnitude above the line of significance was calculated at both the whisking frequency and the theta frequency and compared across animals (Table 1). If this fraction was larger than $5 \%$, as expected by chance, the coherence between whisking and theta rhythm was significant. Seven of eight animals had insignificant coherence across trials, in that the cumulative distribution exceeded $C=0.726$ for $<5 \%$ of the trails (Table 1 ). Critically, a composite analysis that includes all trials in all animals shows that the coherence is insignificant at the $p<0.05$ level (Table 1, All).

As a second means to establish the statistics of the spectral coherence between whisking and the theta rhythm across individual epochs, we calculated the trial-averaged coherence for each animal. Consistent phase locking across epochs would be indicated by a statistically significant value of the magnitude of the coherence after averaging. Four of eight subjects had insignificant trial-averaged coherence. The remaining four subjects had significant, albeit small, trial-averaged coherence (Table 1). Critically, a composite analysis that includes all trials across all animals shows that, on average, the coherence is insignificant (Table 1, All). In summary, we reject the null hypothesis of coherence between exploratory whisking and hippocampal rhythm.

Independent of phase locking, it is possible that whisking and hippocampal theta rhythm activity can modulate each other on the second-to-seconds time scale of individual epochs. To test for this possibility, we performed a linear regression analysis between the peak spectral power in the exploratory whisking band with

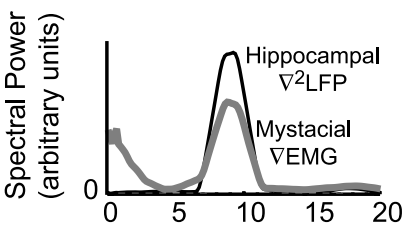

$100 \mu \mathrm{V}$
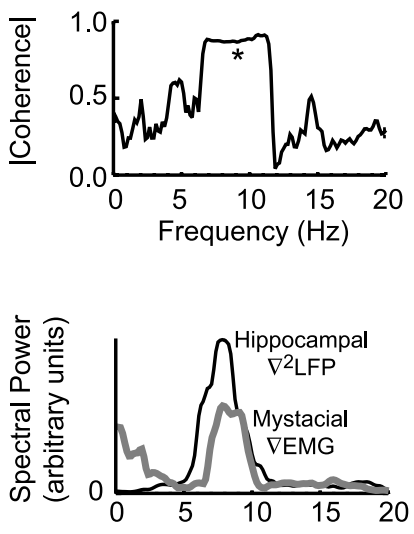

$100 \mu \mathrm{V}$

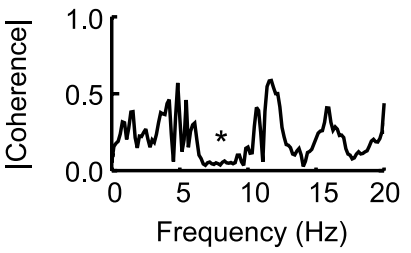

Frequency $(\mathrm{Hz})$
B

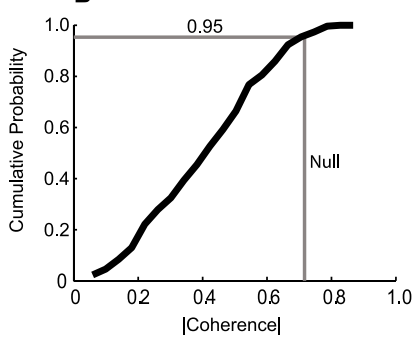

Figure 3. Trial-averaged coherence between whisking and the theta rhythm. $A$, Probability density function (PDF) of coherence magnitudes for a sample animal (F9, $n=264$ ). $B$, Cumulative density function ( $\int P D F$ ) of coherence magnitudes for same animal. The significance line for a $95 \%$ confidence interval is depicted as a horizontal line. The vertical line represents coherence magnitude that $5 \%$ of epochs would be expected to exceed under null hypothesis. The $\int$ PDF would cross the vertical line at $<95 \%$ of the epochs if the number of coherent epochs were significant.

that in the theta rhythm band to determine whether the two signals were statistically correlated (Fig. 4A). Only one of eight animals demonstrated a statistically significant correlation (Fig. $4 B)$ at the $p<0.05$ level. A composite analysis across all epochs in all animals showed that the composite correlation was insignificant $(r=-0.03 ; p=0.013)$. This result indicates that the amplitudes of the two rhythmic signals are independent of each other.

Finally, we revisit the issue of the independence of whisking and the theta rhythm in terms of a sensory lesion, rather than a lesion to the limbic tract (Gray, 1971; Semba and Komisaruk, 1984). Our analysis makes use of the known decrement in frequency of rhythmic whisking in response to lesion of the IoN to 
Table 1. Compendium of trail-by-trial coherence for all animals

\begin{tabular}{llll}
\hline Animal & $\begin{array}{l}\text { Number of } \\
\text { whisking epochs }\end{array}$ & $\begin{array}{l}\text { Trials with coherence above 95\% } \\
\text { significance at } f=f_{\text {whisk }}\end{array}$ & $\begin{array}{l}\text { Trials with coherence above 95\% } \\
\text { significance at } f=f_{\text {theta }}\end{array}$ \\
\hline B1 & 63 & $7.9 \%$ & $6.3 \%$ \\
F2 & 45 & $0.0 \%^{*}$ & $0.0 \%^{*}$ \\
F3 & 38 & $2.6 \%^{*}$ & $2.6 \%^{*}$ \\
F6 & 103 & $2.9 \%^{*}$ & $1.0 \%^{*}$ \\
F7 & 181 & $1.7 \%^{*}$ & $1.7 \%^{*}$ \\
F8 & 100 & $1.0 \%^{*}$ & 0.23 \\
F9 & 264 & $4.9 \%^{*}$ & $0.0 \%^{*}$ \\
K1 & 91 & $1.1 \%^{*}$ & $4.6 \%^{*}$ \\
All & 885 & $3.1 \%^{*}$ & $4.4 \%^{*}$ \\
\hline
\end{tabular}

*Insignificant coherence, i.e., the null hypothesis of significant coherence, based on $p<0.05$, is rejected. ${ }^{* *}$ Insignificant coherence, i.e., the null hypothesis of a significant trial-averaged coherence, based on $p<0.05$, is rejected. ${ }^{a}$ Each epoch is $2 \mathrm{~s}$ in duration with $6 \mathrm{~Hz}<f_{\text {theta }}<9 \mathrm{~Hz}$ and $6 \mathrm{~Hz}<f_{\text {whisk }}<9 \mathrm{~Hz}$.
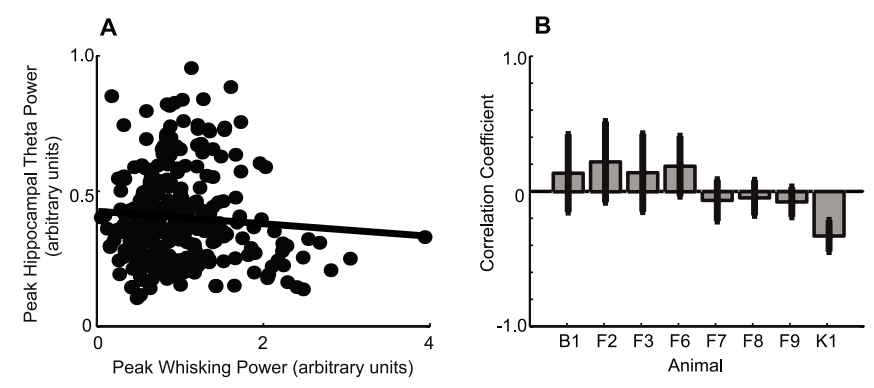

Figure 4. Lack of amplitude correlations between whisking and the theta rhythm. $\boldsymbol{A}$, Peak power of whisking versus peak power of hippocampal signal for animal F9. Each dot represents a $2 \mathrm{~s}$ whisking epoch. The regression line demonstrates that correlation is not statistically significant $(r=-0.08 ; p=0.06)$. $\boldsymbol{B}$, Correlation coefficient and two- $\sigma$ error bars on relationship between peak power of whisking and peak power of hippocampus for each animal subject. There is a significant negative correlation for animal $\mathrm{K} 1$.

block sensory input (Welker, 1964; Berg and Kleinfeld, 2003). We asked whether such a decrement occurs in the hippocampal rhythm as well during exploratory whisking. In agreement with past results, we observed a decrement in the frequency of whisking across four of four animals in which we lesioned the IoN (Table 2). In contrast, only one animal showed a systematic decrease in the frequency of the theta rhythm. This data further supports the independence of the two rhythms.

\section{Discussion}

The present study quantifies the coherence between the rhythmic movement of the rat's vibrissas during exploratory whisking, as inferred from the mystacial electromyogram (Berg and Kleinfeld, 2003) and the hippocampal theta rhythm. Overall, the two rhythms are not locked in a greater percentage of epochs than would be expected by chance (Table 1). This finding reconciles a long-standing disagreement in the literature.

The hippocampal theta rhythm and cortical desynchronization are considered hallmarks of arousal and alerted states (Komisaruk and Olds, 1968; Moruzzi and Magoun, 1995; Berg et al., 2005). In pioneering work, Green and Arduini (1954) recorded from both anesthetized and awake but sessile animals and found that the theta rhythm occurred concomitant with cortical desynchronization. The relationship between these rhythms appears less obvious in drowsy animals (Vanderwolf, 1969). Studies of rhythmic activity in hippocampus during awake behavior were first provided by Grastyan et al. (1959) and Adey et al. (1960), who recorded from cats as they performed learning tasks. Of direct relevance to the present work, Komisaruk and Olds (1968) reported that units in hippocampus were active during bouts of exploratory vibrissa movements, which suggested a link between hippocampal activity and whisking. Furthermore, the hippocampal theta activity and spontaneous vibrissa twitches during paradoxical sleep (Vanderwolf, 1969) appear to be related on a cycleby-cycle basis (Lerma and Garcia-Austt, 1985).

The overlap in frequency range between exploratory whisking and the hippocampal theta frequency makes it tempting to speculate that the two rhythms phase lock during activity. Komisaruk (1970) reported that a "one-to-one correlation was often observed between each vibrissa twitch and each beat of the theta rhythm" although their "rhythmicity are not always phase locked" [Komisaruk (1970), Fig. 5], a result later reaffirmed by Semba and Komisaruk (1984). In complementary work with hamsters, potential coupling of exploratory sniffing with the theta rhythm was studied by Macrides and colleagues (Macrides, 1975; Macrides et al., 1982), who found that "the mystacial musculature often appeared to be correlated with individual hippocampal slow waves at times" yet the timing of the theta rhythm "showed the timing of sniffs relative to peaks or troughs in the slow waves to be steadily drifting." Despite the stated caveats in past work, the notion of a one-to-one correlation between the two rhythms has gained undue acceptance. As quantified in this report, this acceptance is unfounded.

It is important to note that the phase relationship between whisking and the theta rhythm may well depend on the state of the animal. First, recent work showed that the coherence between ongoing cortical activity and vibrissa motion triples when rats are engaged in a reward-based tasks (Ganguly and Kleinfeld, 2004). Second, both past (Adey et al., 1960; Macrides et al., 1982) and

Table 2. Compendium of frequency changes after lesion of the loN

\begin{tabular}{|c|c|c|c|c|}
\hline Animal & $\begin{array}{l}\text { Number of epochs } \\
\text { before incision }^{a}\end{array}$ & $\begin{array}{l}\text { Number of epochs } \\
\text { after incision }^{a}\end{array}$ & $\begin{array}{l}\text { Null hypothesis for equivalent distributions } \\
\text { of whisking frequencies }{ }^{b}\end{array}$ & $\begin{array}{l}\text { Null hypothesis for equivalent distributions } \\
\text { of theta rhythm frequencies }{ }^{b}\end{array}$ \\
\hline F7 & 408 & 343 & Rejected $(p<0.01)$ & Not rejected $(p>0.5)$ \\
\hline F8 & 215 & 179 & Rejected $(p<0.01)$ & Not rejected $(p=0.7)$ \\
\hline F9 & 419 & 295 & Rejected $(p<0.02)$ & Not rejected ( $p=0.8$ ) \\
\hline K1 & 139 & 114 & Rejected $(p<0.01)$ & Rejected $(p<0.01)$ \\
\hline
\end{tabular}

${ }^{a}$ Each epoch is $2 \mathrm{~s}$ in duration.

${ }^{b}$ Kolmogorov-Smirnov test. 
recent (Jones and Wilson, 2005) work suggests the hippocampal theta rhythm and ongoing cortical activity tend to phase lock during a memory task. Thus it is possible that the theta rhythm and whisking will phase lock under certain circumstances, such as when a rat learns to discriminate an object with the vibrissas, as opposed to whisk in air. For example, sniffing is reported to lock to the theta rhythm during a reversal of odor contingency (Macrides, 1975; Macrides et al., 1982), yet is unlikely to be locked to the theta rhythm in the absence of novel odors (Kepecs et al., 2005).

\section{References}

Adey WR, Dunlop W, Hendrix CED (1960) Hippocampal slow waves. Distribution and phase relationships in the course of approach learning. Arch Neurol 3:74-90.

Berg RW, Kleinfeld D (2003) Rhythmic whisking by rat: retraction as well as protraction of the vibrissae is under active muscular control. J Neurophysiol 89:104-117.

Berg RW, Friedman B, Schroeder LF, Kleinfeld D (2005) Activation of nucleus basalis facilitates cortical control of a brainstem motor program. J Neurophysiol 94:699-711.

Buzsaki G (2002) Theta oscillations in the hippocampus. Neuron 33:325-340.

Buzsaki G, Draguhn A (2004) Neuronal oscillations in cortical networks. Science 304:1926-1929.

Carvell GE, Simons DJ, Lichtenstein SH, Bryant P (1991) Electromyographic activity of mystacial pad musculature during whisking behavior in the rat. Somatosens Mot Res 8:159-164.

Ganguly K, Kleinfeld D (2004) Goal-directed whisking behavior increases phase-locking between vibrissa movement and electrical activity in primary sensory cortex in rat. Proc Natl Acad Sci USA 101:12348-12353.

Grastyan E, Lissak K, Madarasz I, Donhoffer H (1959) Hippocampal electrical activity during the development of conditioned reflexes. Electroencephalogr Clin Neurophysiol 11:409-430.

Gray JA (1971) Medial septal lesions, hippocampal theta rhythm and the control of vibrissa movement in freely moving rat. Electroencephalogr Clin Neurophysiol 30:189-197.

Green JD, Arduini AA (1954) Hippocampal electrical activity in arousal. J Neurophysiol 17:533-547.

Hasselmo ME (2005) What is the function of hippocampal theta rhythm? Linking behavioral data to phasic properties of field potential and unit recording data. Hippocampus 15:936-949.

Jarvis MR, Mitra PP (2001) Sampling properties of the spectrum and coherency of sequences of action potentials. Neural Comput 13:717-749.
Jones MW, Wilson MA (2005) Theta rhythms coordinate hippocampalprefrontal interactions in a spatial memory task. PLoS Biol 3:e402.

Kepecs A, Uchida N, Mainen ZF (2005) The sniff as a unit of olfactory processing. Chem Senses 31:167-179.

Kleinfeld D, Berg RW, O'Connor SM (1999) Anatomical loops and their electrical dynamics in relation to whisking by rat. Somatosens Mot Res 16:69-88.

Komisaruk BR (1970) Synchrony between limbic system theta activity and rhythmical behavior in rats. J Comp Physiol Psychol 70:482-492.

Komisaruk BR, Olds J (1968) Neuronal correlates of behavior in freely moving rats. Science 161:810-813.

Lerma J, Garcia-Austt E (1985) Hippocampal theta rhythm during paradoxical sleep. Effects of afferent stimuli and phase relationships with phasic events. Electroencephalogr Clin Neurophysiol 60:46-54.

Macrides F (1975) Temporal relationship between hippocampal slow waves and exploratory sniffing in hamsters. Behav Biol 14:295-308.

Macrides F, Eichenbaum HB, Forbes WB (1982) Temporal relationship between sniffing and the limbic theta rhythm during odor discrimination reversal learning. J Neurosci 12:1705-1717.

Moruzzi G, Magoun HW (1995) Brain stem reticular formation and activation of the EEG. 1949. J Neuropsychiatry Clin Neurosci 7:251-267.

Percival DB, Walden AT (1993) Spectral analysis for physical applications: multitaper and conventional univariate techniques, Chap 6. Cambridge, UK: Cambridge UP.

Robinson TE (1980) Hippocampal rhythmic slow activity (RSA; theta): a critical analysis of selected studies and discussion of possible speciesdifferences. Brain Res 203:69-101.

Semba K, Komisaruk BR (1984) Neural substrates of two different rhythmical vibrissal movements in the rat. Neuroscience 12:761-774.

Thomson DJ (1982) Spectral estimation and harmonic analysis. Proc IEEE 70:1055-1096.

Treves A, Rolls ET (1994) Computational analysis of the role of the hippocampus in memory. Hippocampus 4:374-391.

Vanderwolf CH (1969) Hippocampal electrical activity and voluntary movement in the rat. Electroencephalogr Clin Neurophysiol 26:407-418

Vertes RP (2005) Hippocampal theta rhythm: a tag for short-term memory. Hippocampus 15:923-935.

Welker WI (1964) Analysis of sniffing of the albino rat. Behaviour 12:223-244.

Whishaw IQ, Schallert T (1977) Hippocampal RSA (theta), apnea, bradycardia, and effects of atropine during underwater swimming in the rat. Electroencephalogr Clin Neurophysiol 42:389-396.

Whishaw IQ, Vanderwolf CH (1971) Hippocampal EEG and behavior: effects of variation in body temperature and relation of EEG to vibrissae movement, swimming and shivering. Physiol Behav 6:391-397.

Winson J (1974) Patterns of hippocampal theta rhythm in the freely moving rat. Electroencephalogr Clin Neurophysiol 36:291-301. 